\title{
Unwinding transcriptional bursting
}

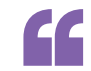

positive supercoiling slows down the rate of transcription elongation
Chong et al. have now shown that, under induced conditions, transcriptional bursting - the observation that transcription occurs in random surges - is regulated through cycles of binding and dissociation of the type II topoisomerase DNA gyrase to a DNA loop in bacteria. Until now, the mechanism underlying transcriptional bursting, which has previously also been observed in eukaryotic cells, was unclear.

Chromosomal structure can influence access of the RNA polymerase to DNA. Positive supercoiling (that is, overwinding of DNA) creates a more compact structure, and the investigators hypothesized that this would affect the rate of transcription. To test this, they developed an in vitro singlemolecule assay using a fluorescent stain that only fluoresces when bound to RNA and that increases in intensity with mRNA length. Incubating DNA templates (which were immobilized to the surface using biotinylated nucleotides) with this dye allowed the rate of transcription to be measured. Using multiple biotinylated nucleotides to inhibit the free rotation of DNA around its surface tether and to enable a build-up of positive supercoils, Chong et al. showed that positive supercoiling slows down the rate of transcription elongation and eventually stops transcription initiation. The addition of gyrase, which releases positive supercoiling, restored transcription elongation rates to the levels of controls and

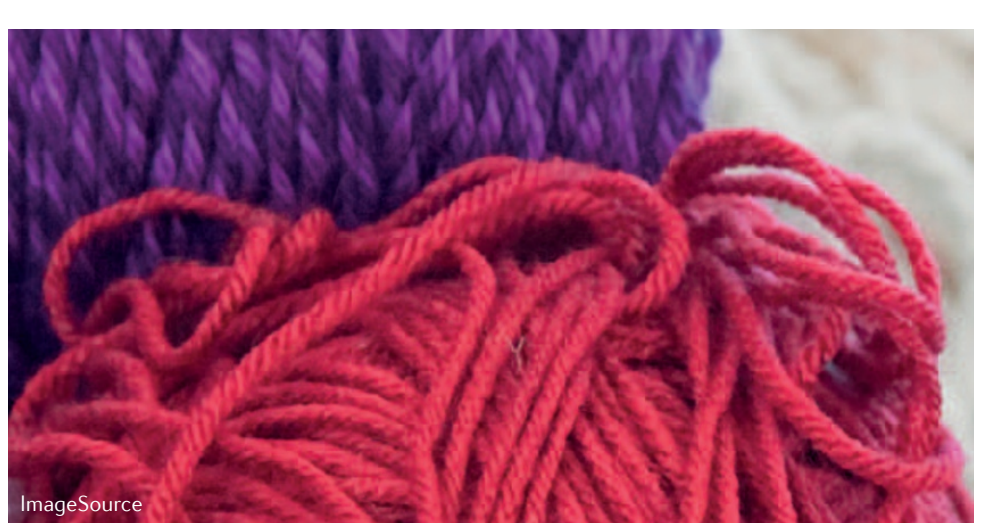

could reinitiate transcription. These results were further confirmed in live Escherichia coli cells.

Although the mechanism in eukaryotic cells is likely to be more complicated, this study highlights the importance of chromosomal architecture on transcriptional bursting.

Isabel Lokody

ORIGINAL RESEARCH PAPER Chong, S. et al. Mechanism of transcriptional bursting in bacteria. Cell 158, 314-326 (2014) 